\title{
Lattice Wess-Zumino Model Simulation with GPUs
}

\author{
Joel Giedt ${ }^{* \dagger}$
}

E-mail: giedtjerpi.edu

\section{Chen Chen}

\section{Eric Dzienkowski}

Department of Physics, Applied Physics and Astronomy, Rensselaer Polytechnic Institute, 110 8th Street, Troy NY 12065 USA

\begin{abstract}
We have numerically evaluated the one-loop counterterms for the four-dimensional Wess-Zumino model formulated on the lattice using Ginsparg-Wilson fermions of the overlap (Neuberger) variety, together with an auxiliary fermion (plus superpartners), such that a lattice version of $U(1)_{R}$ symmetry is exactly preserved in the limit of vanishing bare mass. We have confirmed previous findings by other authors that at one loop there is no renormalization of the superpotential in the lattice theory, but that there is a mismatch in the wavefunction renormalization of the auxiliary field. We studied the range of the Dirac operator that results when the auxiliary fermion is integrated out, and showed that localization does occur, but that it is less pronounced than the exponential localization of the overlap operator. We also presented preliminary simulation results for this model, and outline a strategy for nonperturbative improvement of the lattice supercurrent through measurements of supersymmetry Ward identities. Related to this, some benchmarks for our graphics processing unit code were provided. Our simulation results found a nearly vanishing vacuum expectation value for the auxiliary field, consistent with approximate supersymmetry at weak coupling.
\end{abstract}

The XXVIII International Symposium on Lattice Field Theory, Lattice2010

June 14-19, 2010

Villasimius, Italy

\footnotetext{
* Speaker.

$\dagger$ Supported in part by the Dept. of Energy, Office of Science, Office of High Energy Physics, Grant No. DE-FG0208ER41575 as well as Rensselaer faculty development funds.
} 


\section{Introduction}

One would like to have a general method for studying strongly coupled supersymmetric field theories with lattice techniques. This is because nonperturbative dynamics play an important role in the theory of supersymmetry breaking and its transmission to the visible sector of particle physics. In this proceedings we examine one such general method, which involves a fine-tuning of bare lattice parameters, after having restricted the number of counterterms using lattice symmetries. At the same time, we perform detailed numerical studies of a lattice formulation that was studied by several groups a few years ago $[1,2,3,4,5,6]$. We will highlight some interesting features of that model and present some new results regarding locality of the lattice Dirac operator and the degree of explicit supersymmetry breaking that occurs. This proceedings report touches on aspects that are discussed in much more detail in our recent article [7].

\subsection{Fine-tuning}

Four-dimensional supersymmetric models on the lattice ${ }^{1}$ generically require fine-tuning of counterterms. This is to be contrasted with lower dimensional theories where lattice symmetries can eliminate the need for such fine-tuning; see [11] for further details. The one known fourdimensional exception is pure $\mathscr{N}=1$ super-Yang-Mills using Ginparg-Wilson fermions; the domain wall variety has been the subject of past [14] and recent $[15,16,17,18,10,19]$ simulations. Clearly we would like to go beyond pure $\mathscr{N}=1$ super-Yang-Mills. Recently it was proposed [20] that an acceptable amount of fine-tuning could be efficiently performed using a multicanonical Monte Carlo [21] simulation together with Ferrenberg-Swendsen reweighting [22, 23, 24] in a large class of theories; see also [10]. In this approach it is necessary to design the multicanonical reweighting function. We had suggested beginning at weak coupling on small lattices, using the one-loop perturbative counterterms as initial conditions to an iterative search approach. The present article reports numerical results for one-loop calculations in lattice perturbation theory that are designed to locate this starting point. Ironically we find that for the lattice theory that is studied here, the one-loop counterterms are entirely wavefunction renormalization, and that the logarithmically divergent parts match for the scalar and fermion. As a result, the initial condition for the iterative search is equivalent to starting with the tree-level action, since it is just a rescaling of the fields. This lends interest to our simulation results for the action with no fine-tuning, which we report here.

\subsection{The lattice theory}

The theory that we study is the four-dimensional Wess-Zumino model, formulated on the lattice with a variant of overlap (Neuberger) fermions [25]. The goal of the formulation is to impose the Majorana condition and simultaneously preserve the chiral $U(1)_{R}$ symmetry $[1,2,3,4,5,6]$ that is present in the continuum in the massless limit. As will be seen, preserving this symmetry greatly

\footnotetext{
${ }^{1}$ For reviews with extensive references see $[8,9,10,11,12,13]$.
} 
limits the number of counterterms that must be fine-tuned in order to obtain the supersymmetric continuum limit. In addition to overlap fermions, the lattice formulation has auxiliary fermions (plus superpartner fields) that couple to the overlap fermions through the Yukawa coupling, as in [26]. It is possible to integrate out the auxiliary fermions (and superpartner fields), and when one does this a nonanalytic Dirac operator results for the surviving fermionic field. Thus, as has been discussed originally in [1], and at greater length in $[2,5]$ the action is singular once auxiliary fields are integrated out. However, as we describe below, there is a sensible resolution of this singularity by taking the theory to "live" inside a finite box, with antiperiodic boundary conditions in the time direction for the fermions. The singularity of the Dirac operator that this resolves is related to nonpropagating modes in the infinite volume limit; the fact that these are nonpropagating was shown in [6]. However, singularities in the Dirac operator raise the spectre of possible nonlocalities in the continuum limit, as was found in gauge theories with the SLAC derivative [27]. By analogy to that study, we have analyzed the continuum limit of the scalar self-energy and find that it is analytic in $p^{2}$, so that there is no sign of nonlocality. We have also measured the degree of localization of the Dirac operator following the approach of [28]. We find that while there is localization, it is less pronounced than the exponential localization of the overlap operator. In the process of discussing our numerical perturbative results we are able to highlight the divergence structure of this theory, which turns out to be strictly wave function renormalization at one-loop. The wave function renormalization of the fermion and the physical scalar match at one loop in the continuum limit of the lattice expressions; but, the auxiliary scalar has a mismatched wave function counterterm. These findings appeared previously in [1]; thus we confirm those results.

\subsection{Summary of results}

In [7] we began by discussing our perturbative results. We then reviewed correlation functions and renormalization constants that must be measured in the simulations in order to fine-tune the theory. These involve the renormalized supercurrent (the current is renormalized because the symmetry is broken by the lattice regulator). We concluded with preliminary simulation results. In particular, we have developed all of the components for simulations on graphics processing units. Benchmarks that characterize the performance of our code were reported. We measured one broken Ward-Takahashi identity in a simulation and found it to be very small at weak coupling. We argued that this is consistent with the nonrenormalization of the superpotential at one loop in lattice perturbation theory.

\section{Definitions}

\subsection{Continuum}

The Euclidean continuum theory has action

$$
\begin{aligned}
& S=-\int d^{4} x\left\{\frac{1}{2} \chi^{T} C M \chi+\phi^{*} \square \phi+F^{*} F+F^{*}\left(m^{*} \phi^{*}+g^{*} \phi^{* 2}\right)+F\left(m \phi+g \phi^{2}\right)\right\}, \\
& M=\partial+(m+2 g \phi) P_{+}+\left(m^{*}+2 g^{*} \phi^{*}\right) P_{-} .
\end{aligned}
$$




\subsection{Lattice}

We next discuss the lattice action, which is a special case of the formulations of $[1,2]$; we write the lattice action in forms given in $[4,5,6]$. For this, a few lattice derivative operators must be introduced.

$$
\begin{aligned}
A & =1-a D_{W}, \quad D_{W}=\frac{1}{2} \gamma_{\mu}\left(\partial_{\mu}^{*}+\partial_{\mu}\right)+\frac{1}{2} a \partial_{\mu}^{*} \partial_{\mu} \\
D_{1} & =\frac{1}{2} \gamma_{\mu}\left(\partial_{\mu}^{*}+\partial_{\mu}\right)\left(A^{\dagger} A\right)^{-1 / 2} \\
D_{2} & =\frac{1}{a}\left[1-\left(1+\frac{1}{2} a^{2} \partial_{\mu}^{*} \partial_{\mu}\right)\left(A^{\dagger} A\right)^{-1 / 2}\right] \\
D & =D_{1}+D_{2}=\frac{1}{a}\left(1-A\left(A^{\dagger} A\right)^{-1 / 2}\right)
\end{aligned}
$$

where $\partial_{\mu}$ and $\partial_{\mu}^{*}$ are the forward and backward difference operators respectively. Then the lattice action is [5]:

$$
\begin{aligned}
S= & -a^{4} \sum_{x}\left\{\frac{1}{2} \chi^{T} C D \chi+\phi^{*} D_{1}^{2} \phi+F^{*} F+F D_{2} \phi+F^{*} D_{2} \phi^{*}\right. \\
& -\frac{1}{a} X^{T} C X-\frac{2}{a}\left(\mathscr{F} \Phi+\mathscr{F}^{*} \Phi^{*}\right) \\
& +\frac{1}{2} \tilde{\chi}^{T} C\left(m P_{+}+m^{*} P_{-}+2 g \tilde{\phi} P_{+}+2 g^{*} \tilde{\phi}^{*} P_{-}\right) \tilde{\chi} \\
& \left.+\tilde{F}^{*}\left(m^{*} \tilde{\phi}^{*}+g^{*} \tilde{\phi}^{* 2}\right)+\tilde{F}\left(m \tilde{\phi}+g \tilde{\phi}^{2}\right)\right\}
\end{aligned}
$$

Here, the tilded fields are the linear combinations

$$
\tilde{\phi}=\phi+\Phi, \quad \tilde{\chi}=\chi+X, \quad \tilde{F}=F+\mathscr{F}
$$

The fields $\Phi, X, \mathscr{F}$ and their conjugates are auxiliary fields introduced to allow for a lattice realization of the chiral $U(1)_{R}$ symmetry in the $m \rightarrow 0$ limit:

$$
\begin{aligned}
& \delta \chi=i \alpha \gamma_{5}\left(1-\frac{a}{2} D\right) \chi+i \alpha \gamma_{5} X, \quad \delta X=i \alpha \gamma_{5} \frac{a}{2} D \chi \\
& \delta \phi=-3 i \alpha \phi+i \alpha\left[\left(1-\frac{a}{2} D_{2}\right) \phi-\frac{a}{2} F^{*}\right]+i \alpha \Phi \\
& \delta \Phi=-3 i \alpha \Phi+i \frac{a}{2} \alpha\left(D_{2} \phi+F^{*}\right) \\
& \delta F=3 i \alpha F+i \alpha\left[\left(1-\frac{a}{2} D_{2}\right) F-\frac{a}{2} D_{1}^{2} \phi^{*}\right]+i \alpha \mathscr{F} \\
& \delta \mathscr{F}=3 i \alpha \mathscr{F}+i \frac{a}{2} \alpha\left(D_{2} F+D_{1}^{2} \phi^{*}\right)
\end{aligned}
$$

which takes a particularly simple form on the tilded variables:

$$
\delta \tilde{\chi}=i \alpha \gamma_{5} \tilde{\chi}, \quad \delta \tilde{\phi}=-2 i \alpha \tilde{\phi}, \quad \delta \tilde{F}=4 i \alpha \tilde{F}
$$


As noted in [5], we can integrate out the auxiliary fields $X, \Phi, \mathscr{F}$, treating the tilded fields as constant, to obtain the lattice action: ${ }^{2}$

$$
\begin{aligned}
S= & -a^{4} \sum_{x}\left\{\frac{1}{2} \tilde{\chi}^{T} C M \tilde{\chi}-\frac{2}{a} \tilde{\phi}^{*} D_{2} \tilde{\phi}+\tilde{F}^{*}\left(1-\frac{a}{2} D_{2}\right)^{-1} \tilde{F}\right. \\
& \left.+\tilde{F}^{*}\left(m^{*} \tilde{\phi}^{*}+g^{*} \tilde{\phi}^{* 2}\right)+\tilde{F}\left(m \tilde{\phi}+g \tilde{\phi}^{2}\right)\right\} .
\end{aligned}
$$

This is the lattice action Eq. (2.14) of [4] with a notation that interchanges $D_{1} \leftrightarrow D_{2}$, which is equivalent to Eq. (2.22) of [2] for the $k=0$ case, using the identities ${ }^{3}$

$$
\Gamma_{5}=\gamma_{5}\left(1-\frac{a}{2} D\right), \quad \Gamma_{5}^{2}=1-\frac{a}{2} D_{2}, \quad D^{\dagger} D=\frac{2}{a} D_{2} .
$$

The fermion matrix is:

$$
M=\mathbb{D}+m P_{+}+m^{*} P_{-}+2 g \tilde{\phi} P_{+}+2 g^{*} \tilde{\phi}^{*} P_{-}, \quad \mathbb{D}=\left(1-\frac{a}{2} D_{2}\right)^{-1} D_{1}
$$

Furthermore we can integrate out the auxiliary fields $\tilde{F}, \tilde{F}^{*}$ to obtain the action

$$
S=a^{4} \sum_{x}\left\{-\frac{1}{2} \tilde{\chi}^{T} C M \tilde{\chi}+\frac{2}{a} \tilde{\phi}^{*} D_{2} \tilde{\phi}+\left(m^{*} \tilde{\phi}^{*}+g^{*} \tilde{\phi}^{* 2}\right)\left(1-\frac{a}{2} D_{2}\right)\left(m \tilde{\phi}+g \tilde{\phi}^{2}\right)\right\}
$$

This is the action that is used in our numerical simulations. Note that $\left\{\mathbb{D}, \gamma_{5}\right\}=0$. The theory evades the Nielsen-Ninomiya theorem $[29,30]$ by using a Dirac operator that has singularities in the Brillouin zone. However, the form (2.3) is exponentially local, because it uses the overlap operator. Locality after integrating out auxiliary fields was studied in [7], finding that the localization is less than exponential.

When fine-tuning of the lattice action is performed, we must invoke the most general lattice action consistent with symmetries. Since we perform our simulations at $m \neq 0$, this is just the action with all dimension $\leq 4$ operators built out of the physical fields, $\tilde{\phi}$ and $\tilde{\chi}$. We write it here for reference:

$$
\begin{aligned}
S= & a^{4} \sum_{x}\left\{-\frac{1}{2} \tilde{\chi}^{T} C\left(D D+m_{1} P_{+}+m_{1}^{*} P_{-}\right) \tilde{\chi}+\frac{2}{a} \tilde{\phi}^{*} D_{2} \tilde{\phi}\right. \\
& +m_{2}^{2}|\tilde{\phi}|^{2}+\lambda_{1}|\tilde{\phi}|^{4}+\left(m_{3}^{2} \tilde{\phi}^{2}+g_{1} \tilde{\phi}^{3}+g_{2} \tilde{\phi} \tilde{\phi}^{* 2}+\lambda_{2} \tilde{\phi}^{4}+\lambda_{3} \tilde{\phi} \tilde{\phi}^{* 3}+\text { h.c. }\right) \\
& \left.-\tilde{\chi}^{T} C\left(y_{1} \tilde{\phi} P_{+}+y_{1}^{*} \tilde{\phi}^{*} P_{-}\right) \tilde{\chi}-\tilde{\chi}^{T} C\left(y_{2} \tilde{\phi} P_{-}+y_{2}^{*} \tilde{\phi}^{*} P_{+}\right) \tilde{\chi}\right\}
\end{aligned}
$$

A term linear in $\tilde{\phi}$ has been eliminated by the redefinition $\tilde{\phi} \rightarrow \tilde{\phi}+c$ with $c$ a constant. The parameters $m_{2}^{2}$ and $\lambda_{1}$ are real and all other parameters are complex. Whereas in the supersymmetric theory there are four real parameters, in the most general theory we have eighteen real parameters to adjust. Holding $m_{1}$ and $y_{1}$ fixed, we have fourteen real parameters that must be adjusted to obtain

\footnotetext{
${ }^{2}$ Integrating out an auxiliary fermion to obtain the fermionic part of this action was previously noted in [2]. There it was noted that this relates the fermionic action to the one of [26] by a singular field transformation.

${ }^{3}$ We thank A. Feo for explaining this point and providing us with a derivation of these relations.
} 
the supersymmetric limit. The counting can be alleviated somewhat by imposing CP invariance, so that all parameters can be assumed real. Then we have a total of ten parameters. Holding two fixed, we must tune the other eight to achieve the supersymmetric limit. Conducting a fine-tuning in an eight-dimensional parameter space is a daunting task.

On the other hand in the limit $m_{1} \rightarrow 0$ we can impose the $U(1)_{R}$ symmetry (2.6). This restricts the action to

$$
\begin{aligned}
S= & a^{4} \sum_{x}\left\{-\frac{1}{2} \tilde{\chi}^{T} C D D \tilde{\chi}+\frac{2}{a} \tilde{\phi}^{*} D_{2} \tilde{\phi}+m_{2}^{2}|\tilde{\phi}|^{2}+\lambda_{1}|\tilde{\phi}|^{4}\right. \\
& \left.-\tilde{\chi}^{T} C\left(y_{1} \tilde{\phi} P_{+}+y_{1}^{*} \tilde{\phi}^{*} P_{-}\right) \tilde{\chi}\right\}
\end{aligned}
$$

If we hold $y_{1}$ fixed, then only $m_{2}^{2}$ and $\lambda_{1}$ must be fine-tuned. Conducting a search in a twodimensional parameter space, with both coming from bosonic terms, is manageable. The difficult part is that we must extrapolate to the massless fermion limit. Another potential problem is that we impose antiperiodic boundary conditions for the fermion in the time direction, but must impose periodic boundary conditions for the scalar in order for the action to be single-valued on the circle in the time direction. This breaks supersymmetry explicitly by boundary conditions. At finite mass this should be an effect that can be made arbitrarily small by taking the large volume limit. However at vanishing mass, there will be long distance modes that will "feel" the breaking due to boundary conditions. ${ }^{4}$ Thus it is important that we take $T \gg 1 / m a$ as $m$ is sent to zero, where $T$ is the number of sites in the time direction.

\section{References}

[1] K. Fujikawa and M. Ishibashi, Nucl. Phys. B 622 (2002) 115 [arXiv:hep-th/0109156].

[2] K. Fujikawa and M. Ishibashi, Phys. Lett. B 528 (2002) 295 [arXiv:hep-lat/0112050].

[3] K. Fujikawa, Nucl. Phys. B 636 (2002) 80 [arXiv:hep-th/0205095].

[4] M. Bonini and A. Feo, JHEP 0409 (2004) 011 [arXiv:hep-lat/0402034].

[5] Y. Kikukawa and H. Suzuki, JHEP 0502 (2005) 012 [arXiv:hep-lat/0412042].

[6] M. Bonini and A. Feo, Phys. Rev. D 71 (2005) 114512 [arXiv:hep-lat/0504010].

[7] C. Chen, E. Dzienkowski and J. Giedt, Phys. Rev. D 82 (2010) 085001 [arXiv:1005.3276 [hep-lat]].

[8] J. Giedt, Int. J. Mod. Phys. A 21, 3039 (2006) [arXiv:hep-lat/0602007].

[9] J. Giedt, PoS LAT2006, 008 (2006) [arXiv:hep-lat/0701006].

[10] J. Giedt, Int. J. Mod. Phys. A 24 (2009) 4045 [arXiv:0903.2443 [hep-lat]].

[11] S. Catterall, D. B. Kaplan and M. Unsal, Phys. Rept. 484 (2009) 71 [arXiv:0903.4881 [hep-lat]].

[12] A. Feo, Mod. Phys. Lett. A 19 (2004) 2387 [arXiv:hep-lat/0410012].

\footnotetext{
${ }^{4}$ We thank G. Bergner for raising this point.
} 
[13] I. Montvay, Int. J. Mod. Phys. A 17, 2377 (2002) [arXiv:hep-lat/0112007].

[14] G. T. Fleming, J. B. Kogut and P. M. Vranas, Phys. Rev. D 64, 034510 (2001) [arXiv:hep-lat/0008009].

[15] J. Giedt, R. Brower, S. Catterall, G. T. Fleming and P. Vranas, in proceedings Workshop on Continuous Advances in QCD 2008, Minneapolis, Minnesota, 15-18 May 2008, ed. M. Peloso, World Scientific, Singapore, 2008, arXiv:0807.2032 [hep-lat].

[16] M. G. Endres, in proceedings 26th International Symposium on Lattice Field Theory (Lattice 2008), Williamsburg, Virginia, 14-20 Jul 2008, arXiv:0810.0431 [hep-lat].

[17] J. Giedt, R. Brower, S. Catterall, G. T. Fleming and P. Vranas, Phys. Rev. D 79, 025015 (2009) [arXiv:0810.5746 [hep-lat]].

[18] M. G. Endres, Phys. Rev. D 79 (2009) 094503 [arXiv:0902.4267 [hep-lat]].

[19] M. G. Endres, arXiv:0912.0207 [hep-lat].

[20] J. W. Elliott, J. Giedt and G. D. Moore, Phys. Rev. D 78, 081701 (2008) [arXiv:0806.0013 [hep-lat]].

[21] B. A. Berg and T. Neuhaus, Phys. Lett. B 267 (1991) 249.

[22] M. Falcioni, E. Marinari, M. L. Paciello, G. Parisi and B. Taglienti, Phys. Lett. B 108 (1982) 331.

[23] A. M. Ferrenberg and R. H. Swendsen, Phys. Rev. Lett. 61 (1988) 2635.

[24] A. M. Ferrenberg and R. H. Swendsen, Phys. Rev. Lett. 63 (1989) 1195.

[25] H. Neuberger, "Exactly massless quarks on the lattice," Phys. Lett. B 417 (1998) 141 [arXiv:hep-lat/9707022].

[26] M. Luscher, Phys. Lett. B 428 (1998) 342 [arXiv:hep-lat/9802011].

[27] L. H. Karsten and J. Smit, Phys. Lett. B 85, 100 (1979).

[28] P. Hernandez, K. Jansen and M. Luscher, Nucl. Phys. B 552 (1999) 363 [arXiv:hep-lat/9808010].

[29] H. B. Nielsen and M. Ninomiya, Phys. Lett. B 105 (1981) 219.

[30] H. B. Nielsen and M. Ninomiya, Nucl. Phys. B 185 (1981) 20 [Erratum-ibid. B 195 (1982) 541]. 